\title{
Lieux publics, téléphone mobile et civilité
}

Francis Jauréguiberry

\section{Citer ce document / Cite this document :}

Jauréguiberry Francis. Lieux publics, téléphone mobile et civilité. In: Réseaux, volume 16, n90, 1998. Quelques aperçus sur le téléphone mobile. pp. 71-84;

doi : https://doi.org/10.3406/reso.1998.3187

https://www.persee.fr/doc/reso_0751-7971_1998_num_16_90_3187

Fichier pdf généré le 11/04/2018 


\title{
Résumé
}

La nature des réactions à l'usage des téléphones mobiles dans un lieu public permet de qualitativement désigner ce lieu selon un mode de catégorisation inédit. Plus ces réactions seront nombreuses et négatives, et plus la réputation du lieu renverra à une civilité sensible, à une attention partagée, à une atmosphère de sympathie sociale, à une ambiance positivement vécue, bref au plaisir de goûter au lien social en public. Le mode " d'être- ensemble » dans ces lieux relèvera alors, au mieux d'une urbanité exquise et au pire d'une civilité appréciée. À l'inverse, moins les réactions seront nombreuses et impérieuses, et plus la réputation du lieu renverra à sa simple disposition fonctionnelle, à une approche instrumentale de ses services, à une vision utilitariste de ses ressources, à une relation concurrentielle avec ceux qui le fréquentent, bref à la nécessité de les pratiquer selon une logique utilitariste. Le mode " d'être-ensemble » dans ces lieux relèvera alors, au mieux d'un ajustement bien compris, au pire d'une brutalité urbaine.

\begin{abstract}
The nature of reactions to the use of mobile phones in a public place enables us to qualitatively define this place in terms of an original mode of categorization. The more numerous and negative the reactions, the more the reputation of the place will relate to sensitive civility, shared attention, social fellow feeling, and an atmosphere that is experienced positively; in other words, the pleasure of experiencing the social link in public. The mode of " being together " in this place will, at best, be one of delightful urbanity and, at worst, one of appreciated civility. By contrast, the fewer and less imperious the reactions, the more the reputation of the place will relate to its functional nature, to an instrumental approach to its services, a utilitarian view of its resources and a competitive relationship with those who frequent it; in short, a utilitarian logic. Thus, the mode of " being together " in these places will, at best, be one of a well-understood adjustment and, at worst, a case of urban brutality.
\end{abstract}




\section{LEUXX PUBLCS, TÉLÉPHONE MOBILE ET CIVIUTÉ}

Francis JAURÉGUIBERRY 
$\mathrm{R}$ épondre à un appel alors que l'on est en train de déjeuner avec un ami au restaurant ; engager à la terrasse d'un café une conversation avec quelqu'un que personne ne voit comme si, dérangé mentalement, l'on parlait tout seul ; faire fi de la proximité de son entourage dans une file d'attente pour discuter avec un ailleurs que nul ne connaît : autant de situations que les utilisateurs du téléphone mobile doivent vivre, interpréter et contrôler en fonction de ce qui leur paraît relever de la bienséance, de la politesse et du tact. Cette expérience d'ubiquité médiatique étant inédite, aucune règle n'existe en la matière pour guider les conduites. Pour l'instant, chacun fait donc comme il peut et improvise des réponses en situation.

La sédimentation sociale de ces réponses permet cependant de relever quelques constantes significatives. Par exemple la propension, chez les récents utilisateurs, à téléphoner de façon spontanée quel que soit le lieu où ils se trouvent. L'impression qu'ils retirent de leur nouvelle faculté d'ubiquité leur semble en effet tellement positive et agréable qu'ils en oublient bien souvent leur entourage. Cependant, cette phase " égoïste ", où l'individualité médiatique l'emporte sur la civilité proxémique, est dans la plupart des cas transitoire. Rapidement, une prise de conscience apparaît et un ajustement des conduites en fonction de la présence d'autrui est observable. Mais cet ajustement pèse à son tour peu face aux conduites ostentatoires des nouveaux utilisateurs dont le nombre n'a cessé de se multiplier ces derniers mois. La construction progressive d'usages et en particulier de règles d'utilisation en public est sans cesse recouverte par la conduite « égoïste » des nouveaux utilisateurs. De telle sorte qu'un sans gêne de la téléphonie mobile paraît s'installer partout.

La façon qu'ont les utilisateurs des mobiles de s'extraire d'espaces partagés tout en y restant, ce pouvoir qu'ils s'octroient donc "d'y être sans y être », suscite dans leur entourage au mieux une neutralité désabusée ou un détachement amusé, au pire une attitude offusquée ou une réaction hostile. Nous avons pu observer que plus ces réactions sont spontanées et démonstratives, et plus elles sont négatives (1). Elles visent alors à dénoncer l'empiétement médiatique individuel sur l'espace physique public. Non pas que le branché produise une nuisance sonore (sa voix ne porte pas plus que si son interlocuteur était en face de lui), ni même visuelle (bien que son attitude de retrait le fasse penser), mais parce que son attitude rompt " l'être-là », " l'être-ensemble » du lieu et dénonce par là même la forme de civilité qui lui était jusqu'alors associée.

Toutefois, nous verrons que ces réactions négatives ne concernent que certains lieux publics. Il en est d'autres où l'usage des téléphones mobiles semble parfaitement toléré. Pourquoi cette disparité ? A quels facteurs discriminants peut-on se référer pour comprendre la différence de nature de ces réactions selon les lieux ? Je me propose de montrer dans les pages qui suivent comment l'usage du téléphone mobile fonctionne en la matière comme une sorte de révélateur : il est certains

(1) Cet article s'appuie sur une série de recherches menées durant près de quatre ans auprès des premiers utilisateurs du téléphone portatif à Paris, Strasbourg et Toulouse. Ces recherches ont été financées par le CNET et le CRNS, et menées avec la collaboration de François MENARD, Liliane SOCHAKI et Sandy TORRES au sein du SET (Société Environnement Territoire, unité mixte de recherche 5603 du CNRS - Université de Pau). 
lieux qui font subjectivement lien et d'autres pas. Plus le caractère fonctionnel d'un lieu explique la présence de ceux qui le fréquentent, plus l'utilitarisme et l'individualisme y prédominent, et moins l'usage du téléphone mobile a de chance de gêner. A l'inverse, plus la présence dans un lieu public déborde une simple explication fonctionnelle ou utilitariste, plus ce lieu renvoie à une " ambiance », à une « atmosphère » ou à un « climat » positivement vécus, et plus les réactions à l'usage du téléphone mobile risquent d'être négatives.

\section{L'envol du branché}

« La quatrième dimension, vivez tout l'espace en même temps. " « La quatrième dimension, c'est une nouvelle vision du monde. Vous êtes ici et ailleurs. Vous êtes plus près du tout. Simultanément. Vous vivez tout l'espace en même temps ». «A l'ère de la communication, vous êtes présent partout instantanément ». Ces messages publicitaires de France Télécom, associant télécommunications mobiles et don d'ubiquité, ne font qu'exploiter un vieux désir de l'humanité : pouvoir s'affranchir des contraintes spatiales. En rendant médiatiquement proche ce qui est physiquement lointain, les télécommunications nomades semblent effectivement, comme l'indiquent ces publicités, définir une «nouvelle dimension » dans laquelle les échanges, en se déclinant sous le mode de l'instantanéité, paraissent se jouer de la distance. Lorsqu'on interroge de récents utilisateurs de téléphones mobiles, c'est de l'expérience de cette « nouvelle dimension » qu'ils nous parlent spontanément. Toujours décrite sous le mode d'un étonnement positif si ce n'est jouissif, elle renvoie, selon leur propre vocabulaire, à quelque chose de «fantastique », "d'extraordinaire », de « magique » (2).

«C'est un plaisir, c'est un plaisir de jouer ainsi, sur le temps, sur les lieux...»

« D'être en rase campagne et d'appeler n'importe où... Je trouve que ça a un côté magique. Ça fait rêver... Que je puisse appeler le bout du monde alors que je suis n'importe où... Ça me fait rêver... »

« Je me suis senti comme investi d'un nouveau pouvoir. C'était fantastique !»

«C'est presque incroyable de pouvoir causer, comme ça, à 140 sur l'autoroute à un type qui, si ça se trouve, est lui aussi à $140 \ldots »$

« Avant, il fallait être quelque part pour appeler quelqu'un qui devait être quelque part. Avec ça, je peux être partout pour appeler quelqu'un qui peut être partout. C'est extraordinaire, magique ! »

Pour rendre compte de cette nouvelle expérience, les utilisateurs ont souvent recours à une métaphore aérienne. Les appels nomades sont décrits comme autant d'envols délivrant de la nécessité d'être lié à un lieu pour pouvoir communiquer. Le branché se sent léger, comme détaché d'une contrainte qui devient alors relative.

"Le rêve de l'homme, c'est d'être Icare. Et, grâce au mobile, je suis Icare... Je me sens vachement aérien... C'est génial! »

« Vous êtes sur votre téléphone mobile comme sur un balai magique. »

« Ça permet de s'extraire, c'est comme un décollage. Après, on va où on veut... Il n'y a plus d'obstacle. »

" On se sent un peu flotter en l'air, on est dans l'entre-deux, on n'est plus tout à fait sur terre. »

«C'est comme si l'on s'envolait vers son interlocuteur. »

Professionnellement, ce décollage permet au télécommunicant nomade de se libérer de l'obligation d'une présence à son bureau, du moins dans les cas où celleci n'est motivée que par la nécessité d'une permanence téléphonique.

«Il m'est arrivé de passer des aprèsmidi au bureau, parce que $\mathrm{j}$ 'attendais un coup de fil important. Maintenant, je peux très bien décider de sortir, d'aller au café, faire une course pendant ce temps. Ce temps m'est redonné.»

«Pour les rendez-vous téléphoniques, le

(2) Les citations d'usagers apparaissant dans ce texte sont toutes issues d'interviews menées dans le cadre des recherches citées en note 1 . 
mobile c'est la solution idéale. Quelqu'un vous dit : vous téléphonez à $15 \mathrm{~h} \ldots \mathrm{Et}$ vous n'êtes plus obligé d'être à votre bureau à $15 \mathrm{~h}$... Vous êtes en voiture, vous êtes dans la rue, vous êtes n'importe où....»

« Le mobile permet d'arracher du temps à l'astreinte d'être là. Au lieu d'être là, au garde-à-vous, vous pouvez être ailleurs. »

"Ce sont des moments libres qui nous sont lâchés. »

Ces moments « lâchés ", " redonnés ", « arrachés » sont soit réinvestis dans une occupation professionnelle jugée plus rentable (ce qui est la règle), soit (ce qui est beaucoup plus novateur) consacrés à l'aménagement de bulles d'occupations privées durant le temps professionnel sans que ceci soit culpabilisant. Alors que le téléphone fixe permet généralement des parenthèses médiatiques privées dans des lieux professionnels, le téléphone portatif autorise plutôt des parenthèses professionnelles dans des lieux d'élection privée (3).

« Cet outil m'a permis de jouer au golf en semaine. Ce n'est pas rien, ça, tout de même ! Parce que sinon, j'aurais dû rester au bureau, ou alors $\mathrm{j}$ 'aurais fait mon parcours en me culpabilisant de savoir que l'on n'aurait pas pu me joindre pendant quatre heures et demie. »

«C'est vrai que ça déculpabilise d'avoir un téléphone mobile : si on a envie de se prendre une petite demi-heure ou une heure, voire une matinée entière, on sait qu'on pourra être joignable et on n'a plus de scrupules. Ça m'arrive de partir, d'aller voir quelqu'un, de faire des choses qui n'ont rien à voir avec le boulot. C'est vrai que $\mathrm{j}$ 'ai un boulot indépendant, que je peux me le permettre, mais je ne culpabilise absolument pas, parce que je sais que je pourrais être là dans l'instant. Je peux être tout le temps présent, mais à distance. » (4)

\section{Quand le lieu ne fait plus lien}

A destination d'un téléphone fixe, une personne sait où elle appelle mais pas nécessairement sur qui elle va tomber : le téléphone fixe relie avant tout les lieux. A destination d'un téléphone mobile, la même personne est certaine de tomber sur son interlocuteur, mais ne sait généralement plus où celui-ci se trouve : le téléphone mobile relie d'abord des individus. D'une présence potentielle dans un lieu déterminé, on passe à la potentialité d'une présence dans un lieu indéterminé. Pour l'appelant, la relativisation de l'inscription spatiale de l'appelé devient une sorte de préalable à la télécommunication. Il s'en suit une sorte « d'évaporation » de l'interlocuteur nomade (5).

Mais cette abstraction de la localisation physique des télécommunicants nomades ne va pas sans difficulté. Ainsi, une résistance à l'indétermination ubiquitaire est bien décelable dans la banalisation des questions «t'es où ? », « tu m'appelles d'où ? » et dans la généralisation des précisions topologiques par lesquelles, immanquablement, les appelants ouvrent les conversations. Ces échanges sont toutefois réservés aux interlocuteurs se connais-

(3) Sur ce thème, voir Chantal de GOURNAY, 1997.

(4) Il faut évidemment apporter un bémol à cette « libération » : ne peuvent la mettre en œuvre que ceux qui disposent de leur planning, c'est-à-dire, pour l'essentiel, des cadres supérieurs ou des professionnels indépendants. Pour les autres, et en particulier pour les travailleurs itinérants relevant d'une hiérarchie, le même phénomène peut au contraire se solder par un plus grand contrôle. Car, si le téléphone mobile peut relier en liberté, il peut aussi lier en dépendance : lier sous la forme d'une obligation d'écoute constante, lier au point où l'espace-temps privé se trouve colonisé par le professionnel, lier par l'urgence et la nécessité de réagir immédiatement, lier par le stress qui en résulte et qui produit, en définitive, le sentiment d'être dépossédé de son temps. Sur cette nouvelle forme de dépendance, voir Francis JAUREGUIBERRY, 1998b.

(5) Étudiant les utilisateurs des messageries télématiques, Marc GUILLAUME (1987) utilisait l'expression de fading (évanouissement) pour désigner leur expérience. Les branchés des messageries, analysait-il, sont désincarnés : « ils se projettent dans le réseaux comme des spectres ». Les télécommunicants nomades s'apparentent à ces branchés en ce qu'ils sont, comme eux, désincarnés et atopiques. Mais ils s'en écartent en ce qu'ils demeurent statutairement situés par leurs interlocuteurs. Contrairement aux « spectres » des messageries « qui n'engagent dans leurs échanges qu'une fraction (fiction) d'eux-mêmes via un écran déresponsabilisant " (pseudonymes et jeux de rôles), les télécommunicants nomades savent à qui ils ont affaire. Ils se doivent d'assumer leur rang et d'assurer leur face. 
sant déjà bien : les proches (précisément !), parents ou amis, qui ne peuvent s'imaginer lointains que situés. Pour les appels professionnels, le problème se pose différemment : c'est avant tout une fonction qui est appelée. L'interlocuteur est sensé délivrer une ressource ou une information, et sa non-localisation physique ne pose pas de problèmes particuliers, sauf si l'endroit où il se trouve fait lui-même partie de l'information recherchée. (6)

Cette nouvelle donne communicationnelle peut être habilement jouée au profit d'une « disparition présente ». Ainsi par exemple, cette jeune femme qui donne systématiquement le numéro de son téléphone mobile, alors qu'elle réserve à quelques rares personnes le numéro de son téléphone fixe correspondant à son domicile. Elle s'aménage ainsi, comme elle dit, « la liberté d'être là sans être là »: «On ne sait jamais où je suis lorsque je réponds. Il y a ce côté insaisissable, sans adresse. Avec mon téléphone fixe, les gens savent où $\mathrm{j}$ 'habite et que je suis chez moi [lorsque je réponds], ce qui, pour moi, est déjà une atteinte à la liberté. Par contre, si je suis chez moi et que le téléphone mobile sonne, la personne qui m'appelle ne sait pas où je suis. Et ça, ça me plaît beaucoup ! Je crois que j'adore jouer avec ça! »

Il y a donc deux façons « d'être là sans être là ». La première, pour l'instant la plus courante, consiste à avoir un numéro de téléphone fixe associé à un lieu (qu'il soit personnel ou professionnel) et à le faire basculer, par un transfert d'appel, sur son téléphone portatif : ceci revient à être potentiellement chez soi (ou à son bureau) sans y être physiquement. La seconde consiste à avoir un numéro directement associé à un téléphone mobile, ce qui revient à être toujours potentiellement ailleurs que là où l'on est physiquement (7).

Mais employer le mot d'ubiquité pour désigner ce phénomène est purement métaphorique. Les publicitaires, en faisant passer les télécommunications nomades pour de la pure ubiquité, respectent sans doute bien le principe élémentaire des uses and gratifications sur lequel leur stratégie communicationnelle repose : mettre en scène la réalisation d'un désir en y associant un produit ou un service (8). Mais l'ailleurs médiatique, même s'il est perceptible par l'ouïe et accessible par la voix, n'est pas visible et encore moins palpable. Il demeure physiquement hors d'atteinte. L'ubiquité n'est pas réelle mais partielle. La seule comparaison qui tienne entre les deux espaces se situe au strict niveau relationnel : l'ailleurs médiatique du branché est toujours considéré par lui, au moment où il appelle (ou répond), comme plus rentable, plus nécessaire, plus agréable ou plus utile que ce que l'ici physique lui offre.

C'est au demeurant bien la même impression que retirent les témoins de son « envol ». Pour eux, il y a décollement et éloignement du branché, et ce qu'il vit médiatiquement a toujours l'air d'être beaucoup plus important que sa relation avec son environnement physique. C'est en ce sens que le comportement du branché dans un lieu public peut paraître doublement « déplacé ». D'une part, parce qu'il y a déplacement de son attention vers un point inaccessible aux coprésents, et, d'autre part, parce qu'il rompt ainsi leur "être-ensemble », les règles

(6) La nécessité de situer ainsi ses « proches » interlocuteurs mériterait d'être questionnée plus avant. S'agit-il d'une trace sociale laissée par la généalogie physique des rencontres constitutives des liens affectifs ou d'une permanence anthropo-communicationnelle liée à notre condition physique ? Dans le premier cas, on peut imaginer, avec l'actuel développement des rencontres et relations télécommunicationnelles (par exemple sur Internet), un possible abandon, pour certains, de cette nécessité de situer physiquement leurs proches. Nécessité qui, dans le second cas, existera toujours en creux, donc comme un manque.

(7) Il y a évidemment une troisième façon d'être là sans être là. Mais qui, elle, joue sur le différé et non plus sur le direct, et qui correspond plutôt à « n'être pas là tout en étant là »: le répondeur téléphonique. Le service « Présentation du numéro » de France Télécom (possibilité de savoir d'où provient un appel avant de décrocher) est en train de généraliser cette conduite sous sa forme discriminante (filtre). Pour une étude des usages du répondeur téléphonique, voir Pierre-Alain MERCIER, 1997.

(8) Pour une présentation critique des mythes de l'ubiquité, de la convivialité et du progrès produits par les thuriféraires de la société de l'information, voir Gérard CLAISSE, 1997. 
tacites qui étaient associées au lieu.

«Les personnes qui partagent la même salle de restaurant ou de café, je ne les connais pas, je ne les connaîtrai probablement jamais. Mais il y a tout de même, me semble-t-il, quelque chose de commun : on est dans la même situation, on est tous à la même enseigne, on partage un même espace. À partir du moment où une des personnes est en communication téléphonique avec quelqu'un d'autre, elle sort d'une certaine manière de cet espace. Et je trouve que ça perturbe. »

"La personne qu'on a en face et qui sort son téléphone mobile, on est complétement exclu de son monde. Moi, je me sens vraiment exclue quand quelqu'un, qui est dans le même espace que moi, parle avec un téléphone mobile. »

«L'appel en public, c'est une forme de violence. Ça veut dire : “j’ai plus important à faire." »

Le branché, par sa prise de distance ostentatoire, déchire le fragile tissu de sociabilité qui unissait les physiquements présents malgré leur silence et qui habillait leur sentiment d'exister ensemble dans leurs différences. C'est toute une forme de civilité qui menace alors de tomber en lambeaux (9).

\section{Clivage de l'espace et seuil d'incivilité}

Dans son ouvrage consacré au déclin de la culture publique, Richard Senett définie la civilité comme "l'activité qui protège le moi des autres moi, et lui permet donc de jouir de la compagnie d'autrui. Le port du masque est l'essence même de la civilité. Les masques permettent la pure sociabilité, indépendamment des sentiments subjectifs de puissance, de gêne, etc., de ceux qui les portent. La civilité préserve l'autre du poids du moi (10). Pour Senett, la civilité consiste donc avant tout à respecter une distance à autrui, et le port de masques sociaux est son instrument.

Mais le fait de «ne pas peser sur les autres » ne signifie pas pour autant être indifférent envers eux. Cela implique au contraire un subtil mode de prise en compte de leur présence et d'attention à leur égard. Ainsi, analysent Louis Quéré et Dietrich Brezger, «l'inattention civile est une forme de sociabilité, un mode d'organisation de la co-présence. Elle consiste à créer de la distance entre les personnes, à se rendre poliment étrangers les uns aux autres, à s'installer dans une extériorité des uns par rapport aux autres, à instaurer une a-propriété mutuelle »(11). La civilité nécessite donc à la fois distance et attention. Suffisamment de distance pour que l'autonomie de chacun dans sa spécifité soit préservée, et autant d'attention pour que cette distance ne soit pas synonyme d'indifférence ou de déni.

Posant la question de savoir comment se fait cette démonstration de polite estrangement et quel est son support opérationnel, Quéré et Brezger pensent que « le cœur de cet accomplissement est une organisation de perspectives et une adoption d'attitudes » et soulignent que « la gestion du regard et les postures corporelles jouent un rôle essentiel » (12). Il faut évidemment y ajouter la grande importance de l'ouïe (13). Il s'agit de montrer à autrui, par un type de regard (non-focalisé, furtif ou coup d'œil rapide) et d'écoute (flottante

(9) Lors d'interactions entre personnes se connaissant déjà bien (par exemple autour d'une table de café partagée avec des amis), l'irruption de l'ailleurs médiatique peut être perçue comme étant encore plus déplacée. Le branché signifie en effet clairement à ses vis-à-vis que l'urgence de l'appel l'emporte sur l'importance de leur conversation et que l'advenant téléphonique a priorité sur l'étant proxémique. Mais il est aussi plus facile pour lui de se justifier (en expliquant par exemple, qu'étant de garde, il était impossible de ne pas répondre), de s'excuser (en prétextant par exemple avoir oublié d'éteindre son portable) ou même de réparer l'impair à temps (en abrégeant la télécommunication avec une phrase destinée en fait aux co-présents : «excusez-moi, je ne suis pas seul », ou encore, si l'interlocuteur est connu des co-présents, en les faisant participer à la conversation).

(10) SENETT R., 1979, p. 202.

(11) QUERÉ L. et BREZGER D., 1993, p. 91.

(12) QUERÉ L. et BREZGER D., 1993, p. 94.

(13) Sur ce point, voir Jean-François AUGOYARD, 1989. 
et non focalisée), ainsi que par une gestuelle et un mode particulier d'occupation de l'espace, que l'on se fond dans la normalité comportementale d'être en public. Le vécu de cette étrangeté mutuelle attentionnée, fondatrice du caractère public de certains lieux, relève donc, in fine, de la production des apparences et de leur visibilité, autrement dit d'observabilités.

Or, que donne à voir le branché à ceux qui assistent à son « envol »? Non pas une distance attentionnée, mais une absence intentionnée. Le branché fait, en quelque sorte, fi de sa présence publique pour vivre son absence privée. La réciprocité des perspectives, pensée comme condition de partage d'un environnement commun, n'est dès lors plus possible (14). Les préalables à l'échange des perspectives sont en effet inégalement partagés : la perspective du branché est sensitivement dédoublée (ici et ailleurs à la fois) tandis que celle du ou des témoins de son « envol " demeure unique (ici). Certes, ce dédoublement sensitif n'est qu'auditif. Mais on ne regarde pas pareil quand on écoute ailleurs. Nous avons pu observer comment le regard du branché pouvait parfois « accompagner » l'ouie, comme si, par couplage sensitif, le regard en venait lui aussi à se dédoubler. C'est ainsi qu'un branché, tout absorbé auditivement par son ailleurs, peut visuellement en venir à fixer, d'un regard absent, une autre personne présente. Ce détachement peut alors être vécu par cette personne comme une forme de déni, et donc entraîner une réaction négative.

« En appelant comme ça, [le branché] me nie. J'ai l'impression d'être nié. "

«Moi, ce qui me gêne, ce n'est pas le bruit que font ceux qui téléphonent. Ils ne font finalement pas plus de bruit que s'ils discutaient avec une personne qui serait en face d'eux. Ce qui me gêne, c'est de voir quelqu'un qui parle à quelqu'un d'autre, qui est ailleurs et que je ne vois pas. »
« Dans un espace public, à mon avis, ce qui s'y passe doit être visible. Avec le mobile, on sort de cette visibilité, et je trouve que la règle du jeu n'est pas respectée. Voilà ce qui me déplaît. "

"Dans un café, j'ai une représentation des gens qui m'entourent. Une représentation pas vraiment précise, mais je les vois, ils sont en chair et en os, et surtout ils expriment des choses par leur présence et par leurs gestes. Alors qu'avec le téléphone, je ne sens pas ça. Bien sûr il y a des gestes, il y a même de la gesticulation, mais beaucoup de ce qui est vécu par la personne qui téléphone, est happé par la personne avec laquelle il communique. Et ça, ça échappe. »

L'ailleurs étant inaccessible de façon conjointe, l'ici le devient aussi : il perd son statut d'évidence commune, de cadre aux interactions de co-présence productives de son caractère public. C'est dans le vécu de cette perte de l'éprouvé conjointement, dans cette rupture de sociabilité ou, tout au moins, dans sa déstabilisation et relativisation, que ce qui était jusqu'alors vécu comme implicite devient objet d'interrogations et d'ajustements. C'est en ce sens que le téléphone mobile peut être considéré comme un bon révélateur des formes de l'être-ensemble propre aux lieux publics.

\section{Le téléphone mobile comme révélateur d'urbanité}

Le plus petit dénominateur commun des lieux publics, fondateur de leur caractère public, est leur ouverture à tout un chacun. Le libre accès et la mise en visibilité réciproque de ceux qui le fréquentent fondent l'espace public. C'est ainsi que le caractère plus ou moins public d'un lieu peut se mesurer à l'aune de la place qu'un inconnu de passage peut y trouver (15). Par ailleurs, des lieux publics de statut géné-

(14) C'est parce qu'ego pense qu'alter ferait idéalement la même expérience que lui de leur environnement commun s'il était à sa place (et réciproquement) que cet environnement lui (leur) apparaît comme quelque chose de non seulement commun mais aussi partagé (voir Alfred SCHÜTZ, 1987).

(15) JOSEPH, 1984. 
rique égal (« les cafés », « les cinémas », « les gares » en général) se distinguent aussi entre eux par leur plus ou moins grande capacité à physiquement « exposer » les personnes les unes aux autres par des dispositions de mise en visibilité (16).

Mais les lieux publics ne sont pas seulement des endroits permettant de simples expositions, frictions et observations réciproques. Ils sont aussi et en même temps des lieux d'expression d'un " êtreensemble » et d'existence de sociabilités particulières. Chacun fait quotidiennement l'expérience de la «tonalité » ou de « l'ambiance » des lieux publics qu'il fréquente. Ces « atmosphères » peuvent être vécues comme autant de respitations sociales et de vérifications de la diversité du lien social. Les formes de sociabilités propres aux lieux publics reposent certes sur la mise en visibilité et l'observation réciproque, mais elles se réalisent surtout par la participation active et différenciée de chacun au « lien qui unit dans la séparation » (17). Cette participation est inférée à partir d'une « lecture » des cadres particuliers d'interaction dans lesquels "l'êtreensemble »d'un lieu public se donne concrètement à expérimenter (18).

Il est des cas (beaucoup plus fréquents que les discours défaitistes sur la perte de civilité dans les lieux publics ne le laissent entendre) où cette participation est vécue non plus seulement comme une nécessité (fonctionnelle ou utilitaire), mais aussi comme un plaisir. Plaisir d'être en société et de participer ainsi à un être-ensemble qui échappe à la fois à la fusion communautariste des individus et à la fission individualiste du social. Je propose d'appeler ici « urbanité » cette face attractive et positive de l'être-ensemble dans les lieux publics. En dehors de son acception courante, renvoyant au caractère de « ce qui a rapport à la ville », le mot urbanité revêt une seconde signification, devenue un peu désuète, liant politesse, affabilité et savoirfaire courtois. De cette seconde acception, je ne retiens que de la dimension de respect mutuel et de curiosité détachée qu'elle véhicule. J'évacue la connotation mondaine de recherche formelle de raffinements distinctifs qu'elle prend parfois pour mettre l'accent sur la distance attentionnée ou, ce qui revient au même, sur l'inattention polie qu'elle suggère. L'urbanité est le goût de la civilité, la jouissance de l'écart formalisé comme égard envers autrui et regard envers soi.

Spontanément, chacun associe une « ambiance », une « atmosphère » particulière, aux lieux publics qu'il fréquente habituellement. Il s'attend à y rencontrer un mode de comportement " généralement admis » et donc à y faire l'expérience d'une plus ou moins grande urbanité. C'est parce que cette attente existe et qu'elle donne lieu à des comportements ajustés que les lieux publics, par sédimentation, en viennent à avoir une " réputation d'urbanité ». Bien sûr, cette réputation est sans cesse réactualisée et les dissonances que chacun observe entre réputations escomptées et situations vécues rappellent qu'il s'agit bien d'une construction sociale et qu'il n'existe en la matière aucune urbanité naturelle. Mais, en bousculant aussi soudainement et massivement les comportements " généralement admis » dans les lieux publics, les branchés interrogent de façon beaucoup plus ouverte ces « réputations ». Celles-ci sont en quelque sorte mises en demeure de "s'expliquer » alors qu'elles étaient jusqu'alors « exprimées » comme allant de soi. C'est en ce sens que le téléphone mobile fonctionne comme un révélateur d'urbanité : son usage suscite des réactions qui font apparaître ce qui est déstabilisé. Écouter ces réactions, c'est en grande partie écouter les lieux s'exprimer dans leur réputation d'urbanité.

Or, quelle est la nature de ces réactions

(16) Sur les potentialités différentielles de visibilités des espaces publics, voir Grégoire CHELKOFF et Jean-Paul THIBAUD, 1993.

(17) Étienne TASSIN, 1991, p. 33.

(18) Cette lecture se fait elle-même à partir de l'éventail catégoriel dont dispose l'individu (c'est-à-dire de son capital d'images normatives de l'action qui lui permettent de donner un sens à ce qu'il observe). Sur ce point, voir le débat autour des catégorisations sociales d'Harvey SACKS dans Louis QUÉRÉ et al, 1985. 
lorsqu'elles sont ouvertement exprimées ? D'abord et avant tout, il s'agit de réactions négatives. En quatre ans de recherche sur l'utilisation des téléphones mobiles, je n'ai pas recueilli un seul témoignage ni observé une seule situation qui puisse me permettre de rendre compte de réactions positives à l'utilisation publique des téléphones mobiles. Personne, semble-t-il, n'est encore intervenu ouvertement auprès d'un inconnu téléphonant dans un lieu public pour lui manifester sa joie ou, tout au moins, sa satisfaction d'assister à une telle pratique. L'inverse est par contre quotidiennement vérifiable : les protestations et attitudes d'énervement suscitées par la généralisation des appels téléphoniques publics se multiplient. Certes, un assentiment a sans doute moins besoin d'être exprimé qu'une opposition, surtout lorsque la règle du jeu est une inattention polie. Mais il est d'un autre côté plus aisé et agréable d'extérioriser une approbation qu'un désaccord. On peut donc raisonnablement en inférer que l'usage public du téléphone mobile suscite chez ceux qui en sont témoins plus de réticence que d'adhésion, plus de désagrément que de plaisir.

Les réactions négatives ont souvent lieu sous la forme d'une intervention orale, de la plus cinglante ( $" \mathrm{Je}$ suis dans un train ou à la poste ? », « Vous voulez que je prenne des notes ? ») à la plus détachée ( « Ça coûte cher la minute? », « J'étais venu prendre un café... ») en passant par de l'humour ( « Vous lui direz bonjour de ma part»), par de la provocation (« Autrefois, il y avait des panneaux : ne pas cracher par terre ; je me demande s'il ne faudrait pas les restaurer : ne pas téléphoner à moins de dix mètres ») et même par du dédain ( « Moi, je ne supporterai pas d'être sonné comme ça »). Ces réactions négatives peuvent aussi se donner à voir par le passage d'un regard non focalisé à un regard focalisé si ce n'est insistant : il s'agit de faire comprendre au branché, par effet miroir, que la distance attentionnée « généralement admise » dans ces lieux (réputation) a été rompue (19). La plainte peut encore s'exprimer par un froncement de sourcils, par un soupir sonore, par un geste d'exaspération, par une attitude de repli offensé, etc.

Mais ces réactions ne se donnent pas à voir de manière uniforme avec les mêmes densité et intensité quels que soient les lieux publics. Il est des lieux (par exemple certains cafés, les couloirs ou fumoirs des salles de spectacle, les galeries ou musées) où l'usage des téléphones mobiles, bien qu'officiellement toléré, est pratiquement désapprouvé, tandis que d'autres (par exemple les quais de gare, les salles d'embarquement, les rues passantes) où le même usage ne suscite aucune opposition. Des uns aux autres, un facteur discriminant semble présider le rapport entre réactions et lieux fréquentés. L'hypothèse ici défendue est que ce facteur est le degré d'urbanité spontanément associé au lieu, autrement dit sa réputation d'urbanité.

L'ensemble des matériaux que j'ai pu recueillir autour de cette question permet de proposer un mode de catégorisation des lieux publics selon le type de réaction qu'une conduite ostentatoire d'ubiquité médiatique peut susciter : toutes choses égales par ailleurs (et en particulier les dispositions proxémiques), plus les réactions seront nombreuses et négatives, et plus la réputation du lieu renverra à une civilité sensible, à une attention partagée, à une atmosphère de sympathie sociale, à une ambiance positivement vécue, bref à un goût du lien social en public, autrement dit à un fort degré d'urbanité. Le mode «d'être-ensemble » dans ces lieux relèvera alors, au mieux d'une urbanité exquise et au pire d'une civilité appréciée.

Il est difficile de globalement caractériser ces lieux. On peut cependant relever la nature non obligatoire de leur fréquentation : ce n'est ni la nécessité fonctionnelle ni l'obligation statutaire qui expliquent prioritairement la présence de ceux qui s'y trouvent. L'attrait pour l'urbanité qui s'y

(19) Au niveau des regards, il est une forme indirecte d'intervention d'une grande efficacité : le regard approbateur d'un co-présent vers le plaignant. Outre qu'il renforce la plainte, ce regard tiers, lorsqu'il est capté par le branché, rappelle l'être-ensemble distancié du lieu. 
donne à expérimenter paraît plus importante que toute autre explication. Il semble plus facile d'y goûter au bonheur de ce que Georg Simmel appelle la «pure forme de sociabilité », c'est-à-dire «le lien de réciprocité qui flotte en quelque sorte librement entre les individus » (20). La possibilité de s'y arrêter, de rompre avec l'accélération des rythmes et la généralisation de la simultanéité, de se donner le temps de l'attention gratuite selon un principe de plaisir et, pourquoi pas, d'y faire preuve de compassion, est aussi un dénominateur commun. Le café où l'on s'arrête peut être présenté comme l'archétype de ces lieux publics. Et l'on comprend pourquoi l'attitude du branché, qui consiste à réintroduire l'urgence là où l'arrêt s'est publiquement réfugié, peut susciter des réactions négatives $(21)$.

À l'inverse, moins les réactions seront nombreuses et négatives, et plus la réputation du lieu renverra à sa simple disposition fonctionnelle, à une approche instrumentale de ses services, à une vision utilitariste de ses ressources, à une relation concurrentielle avec ceux qui le fréquentent, bref à la nécessité de les pratiquer selon une logique utilitariste. Il s'agit alors moins de lieux que, pour reprendre le terme de Marc Augé (22), de «nonlieux », c'est-à-dire d'endroits n'évoquant pour ceux qui les fréquentent rien d'autre que leur pure fonctionnalité. Le mode «d'être-ensemble » dans ces lieux relèvera alors, au mieux d'un ajustement bien compris, au pire d'une brutalité urbaine.

Téléphoner dans ce type de lieux publics peut alors répondre du désir de se dégager du caractère obligé de leur fréquentation et de s'abstraire de l'ambiance désagréable qui leur est parfois associée. On peut à ce propos faire l'hypothèse qu'un nombre non négligeable des « envols » des branchés s'apparente pour beaucoup à des tentatives d'échapper à une pure détermination fonctionnelle des trajectoires dans la ville. Le téléphone por- table rend "supportable 》 des déplacements inévitables ou une inscription spatiale non désirée. Ces lieux physiques ne faisant plus lien, le lien médiatique fait dès lors figure de lieux. Il en résulte une sorte de cocooning téléphonique (la publicité « gardez le contact avec votre tribu » en est une illustration) d'individus nomades dans un monde fragmenté. Mais la multiplication de ces appels téléphoniques risque en retour d'aggraver la situation en chargeant ces lieux d'une "sous-urbanité » : la satisfaction éprouvée dans l'espace médiatique dédouane en quelque sorte les branchés de tout effort pour " améliorer » l'ambiance de ces lieux.

Les salles d'embarquement dans les aéroports sont d'excellents lieux d'observation de ce phénomène : le confinement dans l'attente semble produire un besoin urgent de téléphoner. Certains de ces appels sont sans doute importants. Mais, lorsqu'on saisit la nature des conversations, beaucoup portent plutôt à sourire : une fois sur deux, l'information «si urgente à délivrer » consiste à dire " qu'on est bien en salle d'embarquement ", «qu'on va bien partir », «qu'il fait gris» et « qu'on arrivera à telle heure ». On peut alors se demander si cette " téléphonie aiguë »n'est pas une façon de tromper l'attente, de ne pas subir la gêne de ne rien faire, d'échapper à l'obligation d'être là, bref de s'extraire médiatiquement d'un environnement négativement perçu. Lorsque ce genre de contagion a lieu, il semble difficile d'y résister : chacun fait de même, ou se plonge dans la lecture de journaux, à tel point qu'il devient presque incongru de se contenter de la présence des autres.

\section{Développement et ajustement des pratiques nomades}

Durant les quatre dernières années, une nette évolution a pu être observée dans les réactions à l'utilisation du téléphone 
mobile en public. D'abord, les téléphones mobiles se sont multipliés (23). Impossible désormais de ne pas assister plusieurs fois par jour à des conversations téléphoniques publiques. Il y a seulement quelques années, l'utilisation d'un téléphone mobile en public restait une quasi-curiosité dont l'originalité était tolérée sans trop de questionnements. Seuls quelques professionnels de l'urgence y avaient en effet recours, et le prestige dont ils bénéficiaient désamorçait les critiques. Puis les hommes d'affaires et ceux qui voulaient en être sont arrivés... Il semble que ce soit davantage le nombre des appels que leur caractère ostentatoire qui ait commencé à susciter des réactions négatives. Ainsi, certains yuppies, confondant distinction et exhibition ont même un temps été tolérés, au même titre qu'un petit scandale peut l'être dans un lieu public (par exemple provoqué par quelqu'un qui s'adresse à la cantonade dans un café) : après tout, la définition de l'espace public (ouvert à tout un chacun) n'en induit-elle pas le risque?

Le seuil de tolérance a cependant très vite été franchi, provoquant de vives réactions. Emportés par leur nouveau pouvoir d'ubiquité, certains branchés ont sans doute un peu trop fait abstraction de la réputation des lieux servant de cadre à leurs appels. Les réponses ne se sont pas fait attendre. Aussi a-t-on vu des curés demander à leurs paroissiens d'éteindre leur téléphone mobile durant les offices religieux, des présidents de parlement rappeler à l'ordre des députés trop assidûment et ouvertement branchés, des professeurs s'étonner de la "démocratisation » des téléphones mobiles en plein amphithéâtre, et l'on ne compte plus les lieux publics (musées, cinémas, salles de spectacle, restaurants, galeries, cafés, etc.) où l'emploi des téléphones mobiles est formellement interdit.

Mais ce sont surtout les regards des branchés sur eux-mêmes qui ont changé : au fur et à mesure que l'usage des téléphones mobiles se généralisait, les bran- chés sont en effet devenus leurs propres spectateurs et... leurs propres plaignants. Ainsi, cet avocat que nous avons interrogé et qui sortait assez souvent de son bureau pour aller, avec quelques dossiers et son téléphone mobile, s'installer à une table de café pour travailler. En cas d'urgence, son secrétariat pouvant le joindre : il pouvait donc traiter calmement ses affaires dans un cadre agréable. Jusqu'au jour où, constatant qu'une autre personne faisait comme lui, il s'est écrié : "Mince alors! Mon café chéri ! Si tout le monde fait pareil, ça ne sera plus un café ! » Les témoignages de ce type peuvent être multipliés : «L'environnement, dans un café, c'est important. J'y passe des coups de fil, c'est vrai. Mais si dix autres personnes font comme moi... C'est plus possible... C'est plus un café, c'est la Bourse ! »; «Qu'est-ce qui fait qu'on a envie de fréquenter tel café et pas tel autre ? Pas seulement le cadre, mais surtout les gens, l'habitude qu'ils ont de se comporter [les uns envers les autres]. Moi, ce que je cherche, c'est à la fois la présence des autres et la tranquilité. Mais s'ils se mettent tous à téléphoner, ça devient un hall de gare ! " Ces témoignages prouvent au moins une chose : il est plus facile de téléphoner (malgré la perception de l'attitude négative de son entourage) que d'assister à la multiplication des appels.

Ce mouvement de distanciation et de prise de conscience, par effet miroir, des branchés envers leurs propres pratiques est observable dans de nouveaux comportements qui consistent à ajuster au mieux un désir individualiste d'ubiquité avec une volonté de ne pas trop violemment rompre la réputation d'urbanité de certains lieux publics. Ces comportements vont de l'apparition de petits signes de politesse visant à maintenir "l'être-ensemble » du lieu malgré un « envol » téléphonique, au refus pur et simple de se servir d'un téléphone mobile. Tous relèvent du rétablissement de la réciprocité des perspectives et nous " parlent » donc de la réputation des lieux. Ceci est par exemple bien observable lors- 
qu'un branché, conscient de gêner son entourage, tente de se faire pardonner par de petits signes explicites (d'impatience en désignant son téléphone mobile ou d'excuse envers les présents). En exprimant ainsi publiquement son embarras, il explicite la réputation du lieu : ici, l'attention à autrui est (encore) précieuse.

Les tactiques de discrétion, la brièveté des appels ou des réponses, les attitudes de retrait sont autant d'indicateurs sur la volonté de préserver l'ambiance de civilité de certains lieux publics. Il en est même où ces comportements, lorsqu'ils sont systématiques, investissent l'espace d'une « sur-valeur » d'urbanité. La déconnexion médiatique (mettre son mobile sur « off ») est alors vécue comme une victoire sur soi-même où sur son environnement professionnel (qui pousse généralement à la connexion permanente) et permet de goûter de façon inédite à l'ici-présent. 
AUGE M. (1992), Non-lieux. Anthropologie de la surmodernité, Paris, Seuil.

AUGOYARD J.-F. (1989), « Du lien social à entendre ", in Le lien social, Actes du XIII colloque de l'AISLF, Genève, pp. 702-717.

CHELKOFF G. et J.-P. THIBAUD (1993), «L'espace public, modes sensibles », Les Annales de la Recherche Urbaine, $\mathrm{n}^{\circ}$ 57/58, pp. 6-16.

CLAISSE G. (1997), L'abbaye des télémythes, Lyon, Aléas.

GOFFMAN E. (1973), La mise en scène de la vie quotidienne, $t$. 2 : Les relations en public, Paris, Minuit.

GOURNAY Ch. de (1997), « C'est personnel... La communication privée hors de ses murs ", Réseaux, n 82/83, pp. 2139.

GUILLAUME M. (1987), « Le carnaval des spectres », Cahiers internationaux de sociologie, volume LXXXII, pp. 73-81.

JAUREGUIBERRY F. (1998a), «Urbanité ou brutalité médiatique ? L'usage des téléphones portables dans les cafés ", Les Annales de la Recherche Urbaine, $\mathrm{n}^{\circ} 77$, pp. 59-62.

- (1998b), «Télécommunications et généralisation de l'urgence », Sciences de la société, $\mathrm{n}^{\circ} 44$, pp. 83-96.
JOSEPH I. (1984), Le passant considérable, Paris, Méridiens-Kliencksieck.

- (1993), «L'espace public comme lieu de l'action ", Les Annales de la Recherche Urbaine, $\mathrm{n}^{\circ}$ 57/58, pp. 211-217.

MERCIER P.-A. (1997), « Dopo Ze Bip... Quelques observations sur les usages du répondeur téléphonique ", Réseaux, ${ }^{\circ}$ 82/83, pp. 41-68.

QUÉRÉ L. et al (1985), Décrire : un impératif?, Paris, EHESS.

QUÉRÉ L. et BREZGER D. (1993), "L'étrangeté mutuelle des passants " Les Annales de la Recherche Urbaine, $\mathrm{n}^{\circ} 57 / 58$, pp. 89-100.

SENNETT R. (1979), Les tyrannies de l'intimité, Paris, Seuil.

SIMMEL G. (1981), Sociologie et épistémologie, Paris, PUF.

SCHÜTZ A. (1987), Le chercheur et le quotidien, Paris, Méridiens-Klincksieck.

TASSIN E. (1991), « Espace commun ou espace public ? L'antagonisme de la communauté et de la publicité », Hermès, $\mathrm{n}^{\circ} 10, \mathrm{pp} .23-37$. 